Short communication

\title{
gDefrag: A graph-based tool to help defragmenting landscapes divided by linear infrastructures
}

\author{
F. Mestre ${ }^{\mathrm{a}, *}$, F. Ascensão ${ }^{\mathrm{b}, \mathrm{c}, \mathrm{d}}$, A.M. Barbosa ${ }^{\mathrm{a}}$ \\ ${ }^{\text {a }}$ CIBIO/InBio, Centro de Investigação em Biodiversidade e Recursos Genéticos, Pólo de Évora, Universidade de Évora, Casa Cordovil $2^{a}$ Andar, Rua Dr. Joaquim Henrique \\ da Fonseca, 7000-890 Évora, Portugal \\ ${ }^{\mathrm{b}}$ CIBIO/InBio, Centro de Investigação em Biodiversidade e Recursos Genéticos (CIBIO), InBio, Universidade do Porto, Portugal \\ 'Centro de Ecologia Aplicada "Professor Baeta Neves" (CEABN), InBio, Instituto Superior de Agronomia, Universidade de Lisboa, Portugal \\ ${ }^{\mathrm{d}}$ Department of Conservation Biology, Estación Biológica de Doñana F(EBD-CSIC), Sevilla, Spain
}

\section{A R T I C L E I N F O}

\section{Keywords:}

Landscape fragmentation

Linear infrastructures

Habitat accessibility

Mitigation

Road ecology

\begin{abstract}
A B S T R A C T
Habitat fragmentation is a major biodiversity threat. Linear infrastructures (e.g. roads) hamper the movement of individuals and cause non-natural mortality. Roadkill hotspots have been used to define priority areas for road effect mitigation, but data availability and reliability is an issue, particularly on wide spatial scales. Additionally, mitigating the whole infrastructure network is unfeasible. Expedite methods are required to address such challenges. We present the gDefrag package, a graph-based approach that builds on habitat value and accessibility after simplifying the landscape as a graph. Its advantages include not requiring roadkill or movement data, and providing effective methods to deliver reliable information, allowing landscape managers to address landscape fragmentation overall. gDefrag prioritizes roads which should be targeted first to defragment the landscape. The software includes a user-friendly manual and currently implements four prioritization criteria: habitat quality, maximum number of inter-habitat paths, overall landscape connectivity, and simultaneously larger and higher-quality habitats.
\end{abstract}

\section{Introduction}

Linear infrastructures, particularly roads, railways or power-lines (hereafter roads), are responsible for significant and deleterious impacts on biodiversity, including non-natural mortality (e.g. roadkill or collision with power-lines) and habitat fragmentation, altering the landscapes into a patchwork of increasingly smaller areas (Borda-de-água et al., 2017; Van Der Ree et al., 2015). Given the exponential expansion of the transportation networks worldwide (Ibisch et al., 2016; Laurance and Balmford, 2013), the negative effects are expected to have a global impact. Particularly in environmentally sensitive regions, road networks can have a profound impact on ecosystems (Laurance and Balmford, 2013), affecting the dynamics and persistence of animal populations (Borda-de-Água et al., 2014, 2011; Ceia-Hasse et al., 2017; Holderegger and Di Giulio, 2010; Silva et al., 2010).

A major purpose of researchers in conservation biology, as well of infrastructure managers, is to identify roads having a higher risk of mortality or that inflict important barrier effects. Those infrastructures are often targeted to be prioritized for implementing mitigation measures (Crawford et al., 2014; e.g. Cureton and Deaton, 2012). This prioritization is typically, but not exclusively (Mimet et al., 2016), based on information from wildlife mortality surveys, assuming that road mortality occurs mostly in areas of higher connectivity (Grilo et al., 2011; Kang et al., 2016). However, mortality hotspots may be incorrectly identified if surveys are not sufficiently frequent (Santos et al., 2015). They may also not indicate the best sites for road mitigation due to earlier population depression caused by road fatalities, thereby decreasing the number of records over time (Eberhardt et al., 2013; Zimmermann Teixeira et al., 2017). Furthermore, species with high road avoidance may be rarely detected in roadkill surveys (Chen and Koprowski, 2016), despite being heavily affected by the barrier effects due to resource inaccessibility (Ito et al., 2013).

Assuming that a larger amount of connected habitat allows the persistence of larger and more resilient populations and communities, one could target the mitigation of transportation networks in order to maximize the accessible habitat, i.e. the amount of reachable habitat from a given location without crossing any major barrier (Eigenbrod et al., 2008). We suggest that road mitigation should first be supported by a broader landscape-level approach, with the goal of connecting areas divided by roads in a way that maximizes the amount of

\footnotetext{
* Corresponding author.

E-mail addresses: fmestre@uevora.pt (F. Mestre), fjascensao@cibio.up.pt (F. Ascensão), barbosa@uevora.pt (A.M. Barbosa).
} 


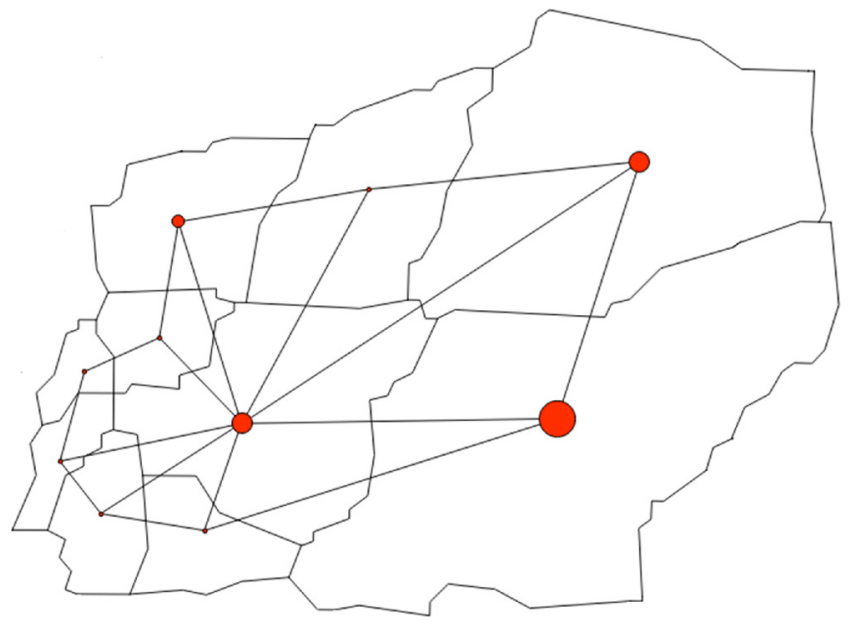

Fig. 1. Nodes (red) and links (black lines) over a landscape fragmented by roads (black polygons).

accessible habitat. The question is how to classify the different roads according to the quantity of quality habitat adjacent to them, prioritizing those that separate patches with higher values. Graph-based tools have increasingly been used in landscape ecology (Foltête et al., 2014; Kang et al., 2016; Urban and Keitt, 2001), and can easily provide a baseline for prioritization. Graph theory applications in landscape ecology usually represent a network of nodes (habitat patches), connected by links, e.g. representing least cost dispersal paths between these patches. Here, we somewhat reverse this reasoning and consider that the nodes are the polygons separated by the infrastructure network, and the links represent (or are crossed by) the infrastructures themselves (Fig.1).

Defining landscapes as spatial graph-like structures presents several advantages, namely they 1 ) effectively summarize the spatial relationships between nodes, 2) facilitate multi-scale analysis, and 3) allow faster computation times (Bergerot et al., 2013; Fortin et al., 2012; Galpern et al., 2011). Moreover, several graph-based connectivity metrics and concepts have already been developed (Brandes, 2001; Freeman, 1978a,b; Plavšić et al., 1993). The approach we propose requires few parameters: the habitat for the focal species or another feature we aim to defragment (based, for instance, on habitat presence, extent, density, suitability or accessibility), and the linear infrastructures network. Such information is available for most regions of the world. Note that one can be interested in defragmenting a particular habitat, e.g. forest cover, or more complex layers such as environmental quality resulting from species distribution models. Likewise, one can integrate different infrastructures simultaneously when considering the polygon areas of interest. Most importantly, this approach allows a fast assessment at the landscape level, and is feasible over any spatial scale. Other software applications exist, e.g. Graphab (Foltête et al., 2012), that translate the landscape as a graph and can be applied in studies related with the impact of linear infrastructures (Girardet et al., 2015). However, this approach is also based upon roadkill data, with all the related issues discussed above.

We hereby present the R package gDefrag (version 0.1 ), containing a set of functions that provide the necessary tools to apply this approach, namely the graph-based prioritization of linear infrastructures for the implementation of mitigation measures, therefore maximizing the defragmentation effectiveness while increasing feasibility and diminishing potential costs.

\section{2. gDefrag workflow}

The package has five functions and a very straightforward workflow, making it an easy-to-use tool for a wide variety of end users (from researchers to infrastructure and landscape managers). The goal is to rank the different components of the linear infrastructure networks (e.g. roads) in order to efficiently prioritize the sections to mitigate while maximizing the accessible habitat and connectivity (Table 1).

The nodes are the spatial polygons defined by the linear infrastructure network (together with the limits of the study area, if necessary). This information is provided by the user, and can be obtained from existing infrastructure layers (e.g. openstreetmaps.org, after correction of topological errors) combined with environmental layers (for the habitat or environmental value of each polygon). Each polygon must have at least one attribute that will be used for prioritizing links, e.g. its area, the amount of available habitat or environmental suitability. The fact that unfavourable landscapes may exist between the linear infrastructure and the closest habitat within the adjacent polygon can be accounted for by the users in the value of this attribute, according to their specific aims. This value can also be weighted by the homogeneity of the habitat or its accessibility from the linear infrastructure. The nodes are created by the function 'node.creation' and are placed inside each polygon. The links connect the nodes (polygons) that are adjacent, separated by linear infrastructures, and are defined using the function 'edge.creation'. Minimum thresholds can be defined for the length of the road separating two polygons, and/or for the area of each polygon, for links to be created connecting them.

The overall workflow can be synthetized in the steps described in Fig. 2. In the following section we will present a worked example resorting to the sample dataset that is made available with the package.

\section{Worked example: prioritizing areas for defragmenting a landscape in a region of the Iberian Peninsula}

\subsection{Load data}

The example that follows uses the sample dataset ('road_P') available with the gDefrag package. It contains a polygon vector map, in which the polygons are delimited by the road network in a region of the Iberian Peninsula (Fig. 3). This small-scaled example provides a simple demonstration of gDefrag capabilities which is relatively fast to compute. As a background raster for obtaining polygons values, we used the percentage of forest cover from Copernicus Global Land Service (Smets et al., 2017). For each polygon we summed the number of cells $(100 \mathrm{~m}$ resolution) with $>30 \%$ forest cover, therefore representing the habitat available for a typical forest species.

\subsection{Node creation}

Next, an object with the nodes (Fig. 4C) was created with the

Table 1

Brief descriptions of the gDefrag functions.

\begin{tabular}{|c|c|}
\hline Function & Description \\
\hline node.creation & Creates the R object with the nodes information, from a layer of spatial polygons separated by infrastructures and characterized by a habitat value. \\
\hline edge.creation & Creates the R object with the links information, connecting the nodes created by the previous function. \\
\hline prioritize & Runs the prioritization algorithm on the links, with one of three methods: 'value', 'between', 'IIC' and 'AWM'. \\
\hline plotgraph & Plots the outputs, including the nodes and links represented according to their values. \\
\hline gDefrag.full & Wrapper function that runs the full process. \\
\hline
\end{tabular}




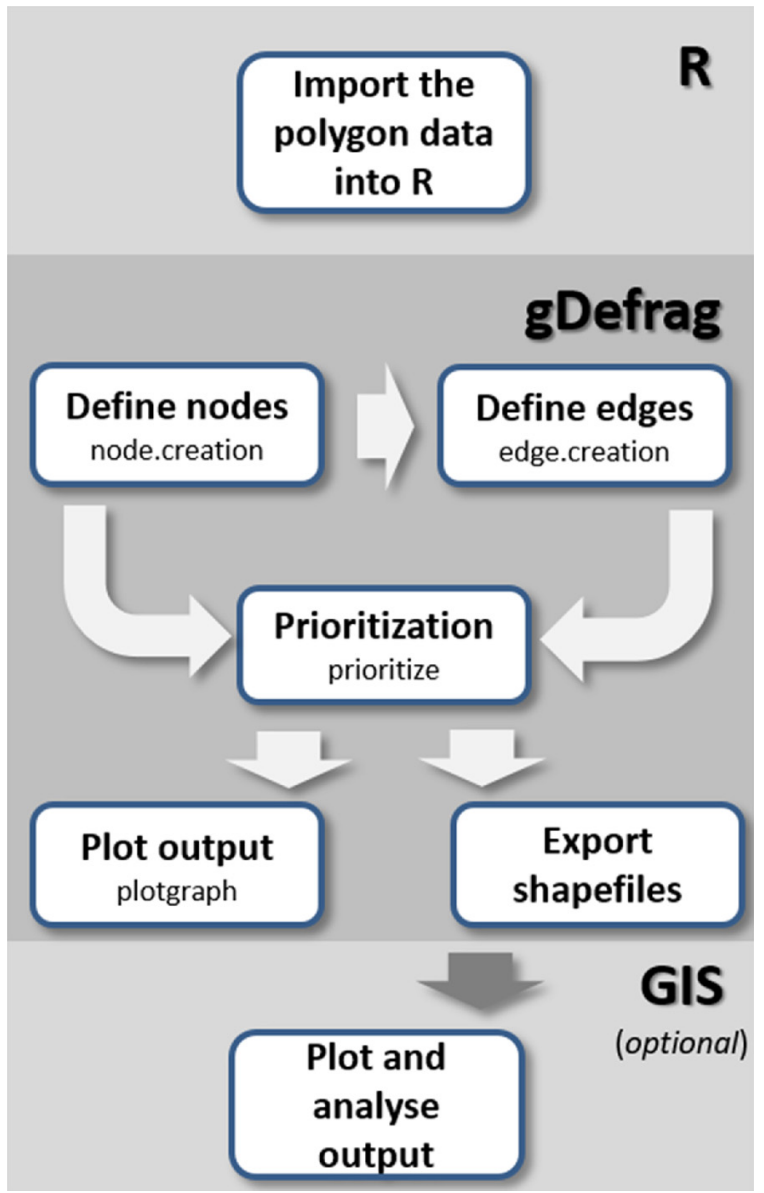

Fig. 2. gDefrag workflow: 1) import polygon data into R; 2) Define nodes; 3) Define links: 4) Prioritization procedure; 5) Plotting and/or exporting output into a GIS.

following code:

out $1<-$ node.creation $\quad$ (land_polyg $=$ road_P, value_col $=$ "forest_sum", scale_nodes $=10$, col_nodes = "pink", cex_labels $=1$ )

\#For the 'Area-Weighted Metric' method

out1_2 $<$ - node.creation (land_polyg = road_P, value_col $=$ "proportion", scale_nodes $=5000000$, col_nodes $=$ "pink", cex_labels $=1$ )

The 'land_polyg' argument requires a 'SpatialPolygonsDataFrame', package 'sp' (Pebesma and Bivand, 2018) object (which can be imported e.g. from a polygon shapefile); 'value_col' requires the name of the column in the polygon data attribute table ('road_P@data') containing the summarized raster information (value); 'scale_nodes' is a graphic argument scaling the node size for a better graphical representation, 'col_node' determines the nodes' colour and 'cex_labels' the size of the nodes labels. The function produces an object of class "SpatialPointsDataFrame" of package 'sp' (Pebesma and Bivand, 2018).

\subsection{Edge creation}

Having the object 'out1' (and 'out1_2'), the links were defined by running the following code (Fig. 4D):

out $2<$ - edge.creation $\quad$ (nodes $=$ out1, land_polyg $=$ road_P, min_length $=0$,

min_pol_area $=0$ )

\#For the "Area-Weighted Metric" method

out2_2 < - edge.creation $\quad$ (nodes $=$ out1_2, land_polyg $=$ road_P, min_length $=0$, min_pol_area $=0$ )

The argument 'nodes' requires the object produced by the function 'node.creation', 'land_polyg' requires the author-input 'SpatialPolygonsDataFrame' ('road_P' in the package sample data), 'min_length' may take the minimum road length of a road separating two polygons to create an edge, and 'min_pol_area' is the minimum polygon area to consider for making a node. The user should give careful consideration to these two last arguments, which have zero as the default value (i.e., no minimum length or area) and can have a relevant impact on the outputs produced in the next function. The function 'edge.creation' creates an object of class 'SpatialLinesDataFrame' of package 'sp' (Pebesma and Bivand, 2018).

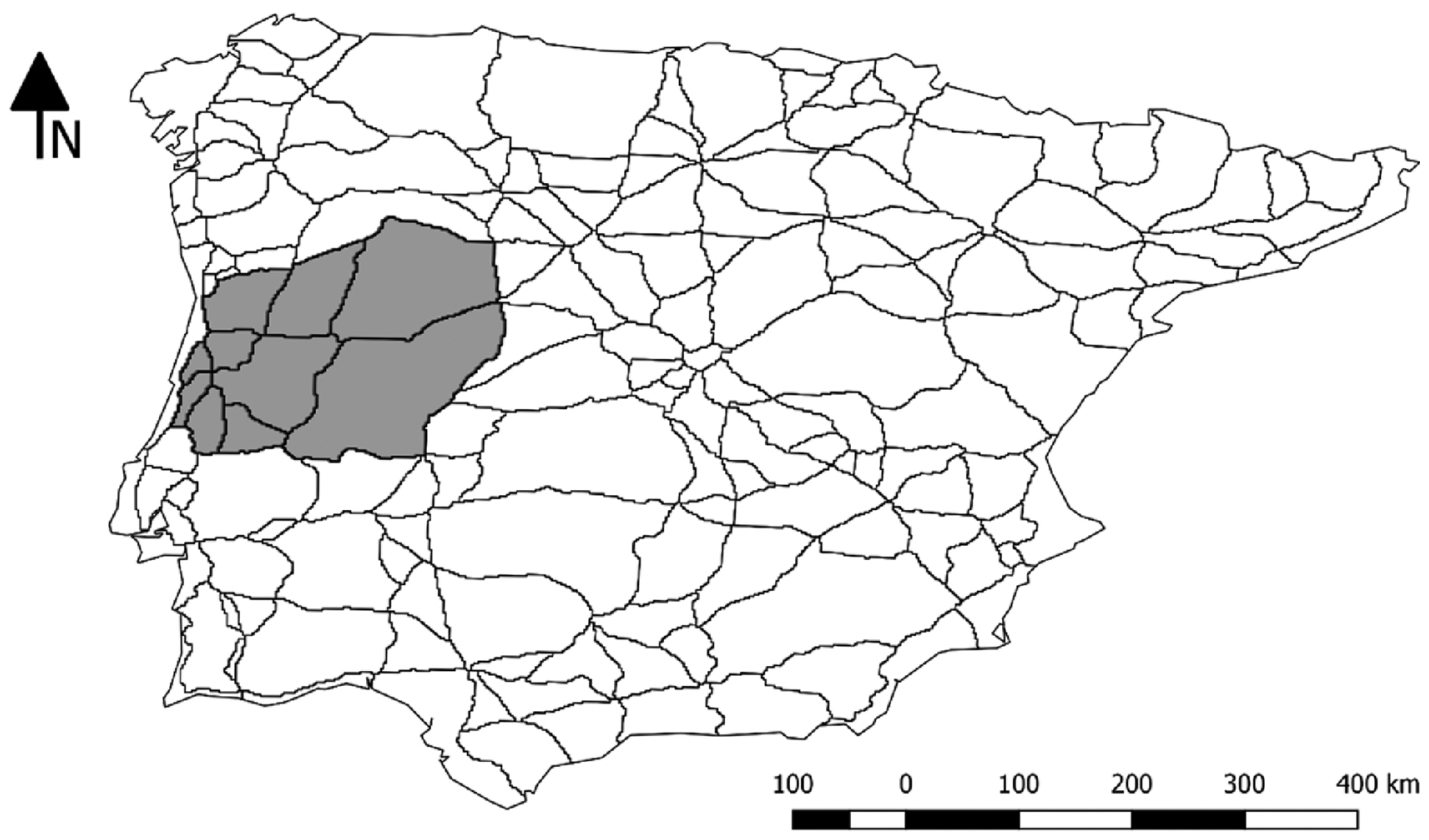

Fig. 3. Iberian Peninsula (SW Europe), with the sub region used as sample dataset highlighted in grey. The lines show the main road network. 


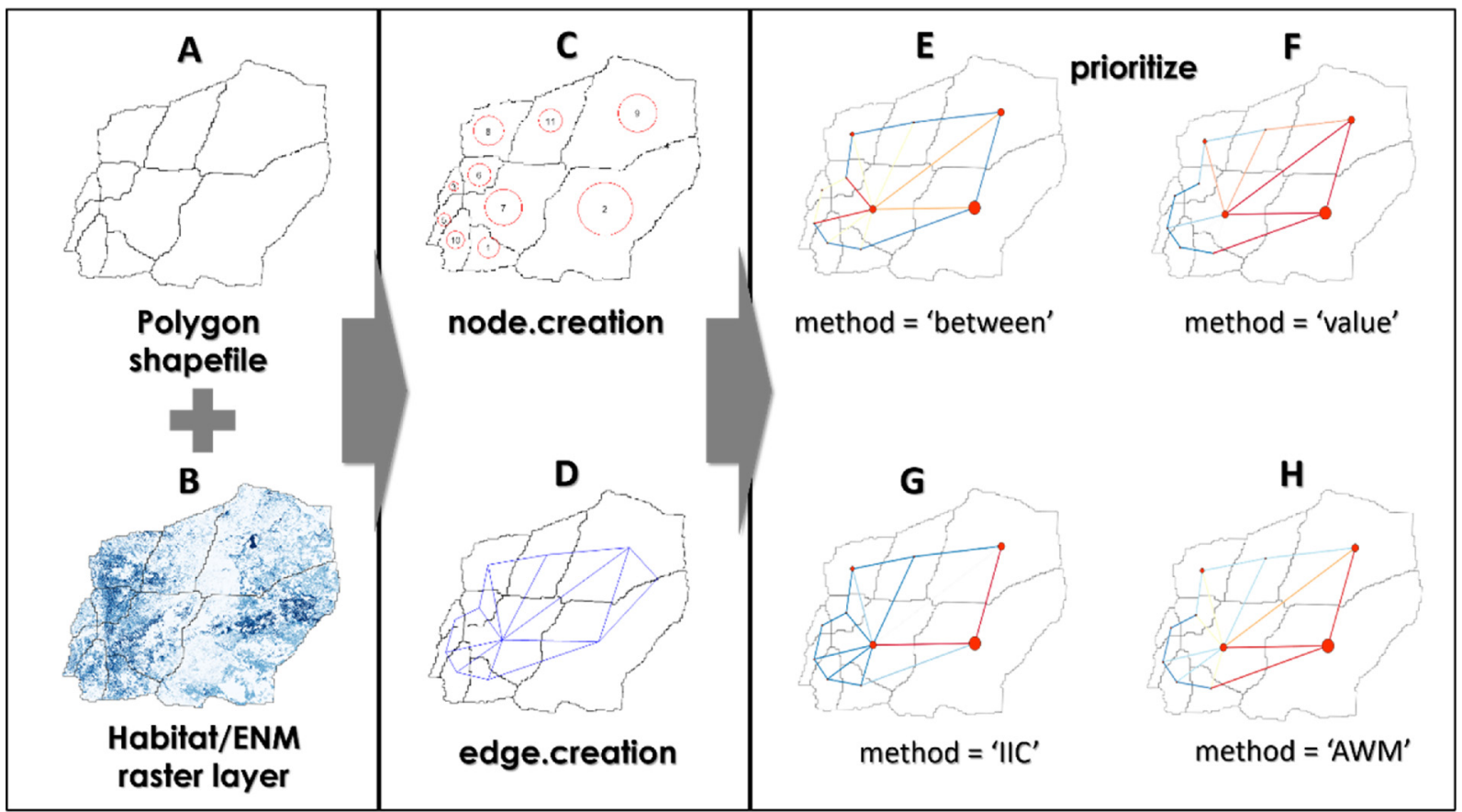

Fig. 4. Sample analysis workflow. Left-hand panel: Combining the polygon shapefile (A) with the raster (B) (e.g. habitat cover, niche model). Mid panel: In gDefrag, create the nodes (C) and the links (D) objects. Right-hand panel: project the prioritization of the links, to the available methods (E, F, G, H). method = 'between' (E) Give priority to links with higher betweenness centrality; method = 'value' (F) - Give priority to nodes maximizing the value of the raster (in this case, connect preferentially nodes with higher forest cover); method = 'IIC' (G) - Integral Index of Connectivity, gives priority to links more relevant to overall connectivity; 'AWM' (H) - Weighted Area Metric, gives priority to larger polygons containing higher habitat quality. Note that the node 4 was not considered in the prioritization computations, considering its small area.

\subsection{Prioritization}

The process of prioritizing the links can be carried out with one of four methods: edge betweenness (Fig. 4E), node value (Fig. 4F), Integral Index of Connectivity (IIC; Fig. 4G) and Area-Weighted Metric (AWM; Fig. $4 \mathrm{H}$ ). The first (method = 'between') prioritizes links that are part of the shortest distance paths (a path is the set of links connecting two nodes; shortest distance refers to the path between two nodes with less links - the topological distance). It is computed by resorting internally to the function 'edge.beetweenness.estimate' of $\mathrm{R}$ package 'igraph' (Csardi and Nepusz, 2018). The method 'value' gives priority to links connecting the nodes with the highest attribute value (which is supplied by the user in the attribute table of the input map). The method 'IIC' gives higher priority to links that are more relevant to the overall graph connectivity, by computing dIIC as described in Pascual-Hortal and Saura (2006). This value is computed for all links, allowing prioritization according to the connectivity value of the links. Finally, the method ' $A W M$ ' prioritizes links based on a combination of size and quality, favoring the defragmentation of larger, higher-quality areas.

The following code produces the output for each of the prioritization methods:

out $3<$ - prioritize $($ nodes $=$ out 1 , edges = out 2 , method = "value")

out $4<$ - prioritize $($ nodes $=$ out 1 , edges $=$ out 2 , method $=$ "between")

out $5<-$ prioritize $($ nodes $=$ out 1, edges $=$ out 2, method $=$ "IIC")

out 6 - prioritize $\quad($ nodes $=$ out1_2, edges $=$ out2_2, method $=$ "AWM")

The outputs provided by this function are depicted in Fig. 4 (bottom panel). The function 'prioritize' creates an object of class
'SpatialLinesDataFrame' of package 'sp' (Pebesma and Bivand, 2018), in which the links (spatial lines) have the priority information in their attribute table.

As an alternative, the complete process can be run using a single wrapper function, 'gDefrag.full', which encloses all others (node.creation, edge.creation, prioritization), but turns the process of creating prioritized links even more straightforward:

out7 < - gDefrag.full (land_polyg = road_P, method = "value", value_col = "forest_sum", main = "Node value-based graph", shape $=$ TRUE)

out8 $<$ - gDefrag.full (land_polyg $=$ road_P, value_col $=$ "forest_sum", method = "IIC", main = "IIC-based graph",shape = TRUE)

out9 < - gDefrag.full (land_polyg = road_P, value_col = "forest_sum", method = "between", main = "Betweenness-based graph", shape $=$ TRUE)

out10 $<$ - gDefrag.full (land_polyg $=$ road_P, value_col $=$ "proportion", method = "AWM", main = "AWM-based graph", shape = TRUE)

The function 'gDefrag.full' produces a list object as an output, including the nodes and the links with their priority values.

\section{Summary results}

Different prioritizing methods prioritize different features of the graph. Betweenness gives more weight to links that are part of more internode shortest paths, thus prioritizing those that maximize the flow between nodes (Fig. 4E). Node value gives more weight to links connecting nodes with higher attribute values. This attribute is user-defined and it can reflect ecological suitability (the output of an ecological niche model), habitat availability (percentage/area of habitat, possibly weighted by accessibility across the road) or available area (polygon 
area) (Fig. 4F). IIC gives more weight to links that, if lost, would have a greatest impact on overall connectivity (Fig. 4G). Finally, the Weighted Area Index gives more weight to larger polygons containing higher habitat quality (Fig. 4H).

\section{Visualization}

The other available function in gDefrag is 'plotgraph', a plotting function to depict the results of the prioritization procedure, although the user can also resort to the possibility of creating a shapefile (argument 'shape' available in all other functions) that can be loaded into a GIS for a more flexible graphical output.

\section{Discussion}

The present package presents an effective and straightforward approach to prioritize areas for defragmenting a landscape. It avoids the aforementioned issues related to wildlife roadkill data, which are often scarce, uneven and of insufficient quality, particularly on wide spatial scales, and therefore might not accurately reflect the relevance of a given site to the focal taxonomic group. We provide a small-scale example in which we used all four prioritization methods that are currently implemented. The results reflect these different approaches, giving more weight to the links configuration (betweenness and IIC) or to the node arguments (value and AWM). 'Betweenness' prioritizes links promoting the flow through the graph, while 'IIC' favours links connecting the graph internally to the farthest nodes. On the other hand, 'value' prioritizes links connecting nodes with the higher user-defined value and the 'AWM' weights this by the polygons' area.

We have shown that gDefrag is an effective tool, providing methods to prioritize links to be created, reducing the fragmentation on a landscape traversed by a road network. It is an easy-to-use, scale-independent approach that allows the selection of sites to break the barrier effect of roads, with the advantage of not requiring roadkill data. Our approach provides a general idea of what are the most strategic portions of linear infrastructures for defragmenting landscapes, even over very wide scales (e.g. regional or continental). Then, finerscale analyses integrating the composition and configuration of the landscape can also be developed, in order to efficiently prioritize the sections to mitigate.

\section{Acknowledgments}

F.M. was supported by the Fundação para a Ciência e Tecnologia (FCT), Portugal, project StateShifts (ref.: PTDC/AAG-MAA/3764/ 2014). F.A. was supported through a FCT post-doctoral grant (SFRH/ BPD/ 115968/2016). A.M.B. was supported by FCT and FEDER/ COMPETE 2020 through contract and exploratory project IF/00266/ 2013/CP1168/CT0001.

\section{Appendix A. Supplementary data}

Supplementary material related to this article can be found, in the online version, at doi:https://doi.org/10.1016/j.ecolmodel.2018.10. 012.

\section{References}

Bergerot, B., Tournant, P., Moussus, J.P., Stevens, V.M., Julliard, R., Baguette, M., Foltête, J.C., 2013. Coupling inter-patch movement models and landscape graph to assess functional connectivity. Popul. Ecol. 55, 193-203. https://doi.org/10.1007/s10144012-0349-y.

Borda-de-água, L., Barrientos, R., Beja, P., Pereira, H.M., 2017. Railway Ecology. Springerhttps://doi.org/10.1007/978-3-319-57496-7.

Borda-de-Água, L., Grilo, C., Pereira, H.M., 2014. Modeling the impact of road mortality on barn owl (Tyto alba) populations using age-structured models. Ecol. Modell. 276, 29-37. https://doi.org/10.1016/j.ecolmodel.2013.12.022.

Borda-de-Água, L., Navarro, L., Gavinhos, C., Pereira, H.M., 2011. Spatio-temporal impacts of roads on the persistence of populations: analytic and numerical approaches. Landsc. Ecol. 26, 1-13. https://doi.org/10.1007/s10980-010-9546-2.

Brandes, U., 2001. A faster algorithm for betweenness centrality. J. Math. Sociol. 25, 163-177. https://doi.org/10.1080/0022250X.2001.9990249.

Ceia-Hasse, A., Borda-de-Água, L., Grilo, C., Pereira, H.M., 2017. Global exposure of carnivores to roads. Glob. Ecol. Biogeogr. 26, 592-600. https://doi.org/10.1111/geb. 12564.

Chen, H.L., Koprowski, J.L., 2016. Barrier effects of roads on an endangered forest obligate: influences of traffic, road edges, and gaps. Biol. Conserv. 199, 33-40. https:// doi.org/10.1016/j.biocon.2016.03.017.

Crawford, B.A., Maerz, J.C., Nibbelink, N.P., Buhlmann, K.A., Norton, T.M., Albeke, S.E., 2014. Hot spots and hot moments of diamondback terrapin road-crossing activity. J. Appl. Ecol. 51, 367-375. https://doi.org/10.1111/1365-2664.12195.

Csardi, G., Nepusz, T., 2018. igraph: Network Analysis and Visualization.

Cureton, J.C., Deaton, R., 2012. Hot moments and hot spots: identifying factors explaining temporal and spatial variation in turtle road mortality. J. Wildl. Manage. 76, 1047-1052. https://doi.org/10.1002/jwmg.320.

Eberhardt, E., Mitchell, S., Fahrig, L., 2013. Road kill hotspots do not effectively indicate mitigation locations when past road kill has depressed populations. J. Wildl. Manage. 77, 1353-1359. https://doi.org/10.1002/jwmg.592.

Eigenbrod, F., Hecnar, S.J., Fahrig, L., 2008. Accessible habitat: an improved measure of the effects of habitat loss and roads on wildlife populations. Landsc. Ecol. 23, 159-168. https://doi.org/10.1007/s10980-007-9174-7.

Foltête, J.-C., Clauzel, C., Vuidel, G., 2012. A software tool dedicated to the modelling of landscape networks. Environ. Model. Softw. 38, 316-327. https://doi.org/10.1016/ J.ENVSOFT.2012.07.002.

Foltête, J.-C., Girardet, X., Clauzel, C., 2014. A methodological framework for the use of landscape graphs in land-use planning. Landsc. Urban Plan. 124, 140-150. https:// doi.org/10.1016/j.landurbplan.2013.12.012.

Fortin, M.J., James, P.M.A., MacKenzie, A., Melles, S.J., Rayfield, B., 2012. Spatial statistics, spatial regression, and graph theory in ecology. Spat. Stat. 1, 100-109. https://doi.org/10.1016/j.spasta.2012.02.004.

Freeman, L.C., 1978a. Centrality in social networks. Soc. Netw. 1, 215-239. https://doi. org/10.1016/0378-8733(78)90021-7.

Freeman, L.C., 1978b. Centrality in social networks conceptual clarification. Soc. Netw. 1 , 215-239. https://doi.org/10.1016/0378-8733(78)90021-7.

Galpern, P., Manseau, M., Fall, A., 2011. Patch-based graphs of landscape connectivity: a guide to construction, analysis and application for conservation. Biol. Conserv. https://doi.org/10.1016/j.biocon.2010.09.002.

Girardet, X., Conruyt-Rogeon, G., Foltête, J.-C., 2015. Does regional landscape connectivity influence the location of roe deer roadkill hotspots? Eur. J. Wildl. Res. 61, 731-742. https://doi.org/10.1007/s10344-015-0950-4.

Grilo, C., Ascensão, F., Santos-Reis, M., Bissonette, J.A., 2011. Do well-connected landscapes promote road-related mortality? Eur. J. Wildl. Res. 57, 707-716. https://doi. org/10.1007/s10344-010-0478-6.

Holderegger, R., Di Giulio, M., 2010. The genetic effects of roads: a review of empirical evidence. Basic Appl. Ecol. 11, 522-531.

Ibisch, P.L., Hoffmann, M.T., Kreft, S., Pe'er, G., Kati, V., Biber-Freudenberger, L. DellaSala, D.A., Vale, M.M., Hobson, P.R., Selva, N., 2016. A global map of roadless areas and their conservation status. Science 354, 1423-1427. https://doi.org/10. 1126 /science.aaf7166.

Ito, T.Y., Lhagvasuren, B., Tsunekawa, A., Shinoda, M., Takatsuki, S., Buuveibaatar, B., Chimeddorj, B., 2013. Fragmentation of the habitat of wild ungulates by anthropogenic barriers in Mongolia. PLoS One 8, e56995.

Kang, W., Minor, E.S., Woo, D., Lee, D., Park, C.-R., 2016. Forest mammal roadkills as related to habitat connectivity in protected areas. Biodivers. Conserv. 25, 2673-2686. https://doi.org/10.1007/s10531-016-1194-7.

Laurance, W.F., Balmford, A., 2013. A global map for road building. Nature 495, 308-309. https://doi.org/10.1038/495308a.

Mimet, A., Clauzel, C., Foltête, J.-C., 2016. Locating wildlife crossings for multispecies connectivity across linear infrastructures. Landsc. Ecol. 31, 1955-1973. https://doi. org/10.1007/s10980-016-0373-y.

Pascual-Hortal, L., Saura, S., 2006. Comparison and development of new graph-based landscape connectivity indices: towards the priorization of habitat patches and corridors for conservation. Landsc. Ecol. 21, 959-967. https://doi.org/10.1007/s10980006-0013-z.

Pebesma, E., Bivand, R., 2018. sp: Classes and Methods for Spatial Data.

Plavšić, D., Nikolić, S., Trinajstić, N., Mihalić, Z., 1993. On the Harary index for the characterization of chemical graphs. J. Math. Chem. 12, 235-250. https://doi.org/ 10.1007/BF01164638.

Santos, S.M., Marques, J.T., Lourenç, A., Medinas, D., Barbosa, A.M., Beja, P., Mira, A., 2015. Sampling effects on the identification of roadkill hotspots: implications for survey design. J. Environ. Manage. 162, 87-95. https://doi.org/10.1016/j.jenvman. 2015.07.037.

Silva, J.P., Santos, M., Queirós, L., Leitão, D., Moreira, F., Pinto, M., Leqoc, M., Cabral, J.A., 2010. Estimating the influence of overhead transmission power lines and landscape context on the density of little bustard Tetrax tetrax breeding populations. Ecol. Modell. 221, 1954-1963. https://doi.org/10.1016/j.ecolmodel.2010.03.027.

Smets, B., Buchhorn, M., Lesiv, M., Tsendbazar, N.-E., 2017. Copernicus Global Land Operations "Vegetation and Energy.

Urban, D., Keitt, T., 2001. Landscape connectivity: a graph-theoretic perspective. Ecology 82, 1205-1218. https://doi.org/10.1890/0012-9658(2001)082[1205:LCAGTP]2.0. $\mathrm{CO} ; 2$.

Van Der Ree, R., Smith, D.J., Grilo, C., 2015. Handbook of Road Ecology, Handbook of Road Ecology. John Wiley \& Sons, Ltd, Chichester, UK. https://doi.org/10.1002/ 9781118568170.

Zimmermann Teixeira, F., Kindel, A., Hartz, S.M., Mitchell, S., Fahrig, L., 2017. When road-kill hotspots do not indicate the best sites for road-kill mitigation. J. Appl. Ecol. 54, 1544-1551. https://doi.org/10.1111/1365-2664.12870. 